\title{
Multi-agent smart environments
}

\author{
Diane J. Cook \\ School of Electrical Engineering and Computer Science, Washington State University, USA \\ E-mail:cook@eecs.wsu.edu
}

\begin{abstract}
Research in pervasive computing and ambient intelligence has catapulted smart environments to the forefront of many research labs. While tremendous strides have been made toward realizing the dream of designing environments that act as intelligent agents, the size of the problem creates issues that still need to be addressed by researchers. One such issue is the role of multi-agent systems in smart environments. In this article we discuss the role of multi-agent research in the context of smart environments and survey current research in the area. In addition, we pose several challenges to be tackled in this dynamic and complex area of research.
\end{abstract}

Keywords: Multiple residents, multi-agent systems, negotiation, tracking

\section{Introduction}

While individuals have dreamed about living in smart environments, only recently has researched advanced to the point where this dream can become a reality. Building on the definition of an intelligent agent as envisioned by Russell and Norvig [17], we can view an intelligent environment as an agent that perceives the state of the environment using sensors and acts on the environment using controllers (see Fig. 1). The actions are selected in such a way that the resident's experience in the environment is optimized for dimensions such as comfort, productivity, or safety [1].

Because the software architecture for a smart environment is so complex, and because there may be multiple entities (e.g., residents) that the environment is serving, a smart environment can be viewed as a multi-agent system. The purpose of this paper is to provide an overview of the role of multi-agent research in the context of smart environments and to pose some challenges that researchers in this field can tackle.

Before we can survey the field of multi-agent research for smart environments, we need to first identify what we are referring to as an "agent" in the context of a smart environment. From one perspective, an agent can refer to a software component that contributes to the software architecture of the smart envi- ronment. From another perspective, we can argue that a smart environment housing multiple residents is a natural multi-agent setting. In the second case, the residents themselves can be viewed as intelligent agents. In addition, software needs to be designed to reason about and interact with each resident, thus transforming the software itself into a multi-agent system.

Resulting from these multiple perspectives on multi-agent systems for software environments, we see four major directions in multi-agent research that are currently being pursued for smart environments. These directions are highlighted in Fig. 2. As the figure shows, there is active research in four major areas. The first focuses on multi-agent software design for smart environments. The others provide solutions to challenges that arise when multiple residents are in a smart environment: tracking multiple residents, profiling activities and behaviors for multiple residents, and negotiating the needs and preferences of multiple agents.

\section{Multi-agent smart environment architectures}

Complex systems such as smart environments require a large number of software components. Each of these components, when containing some autonomy and driven by an intelligent design, can be 


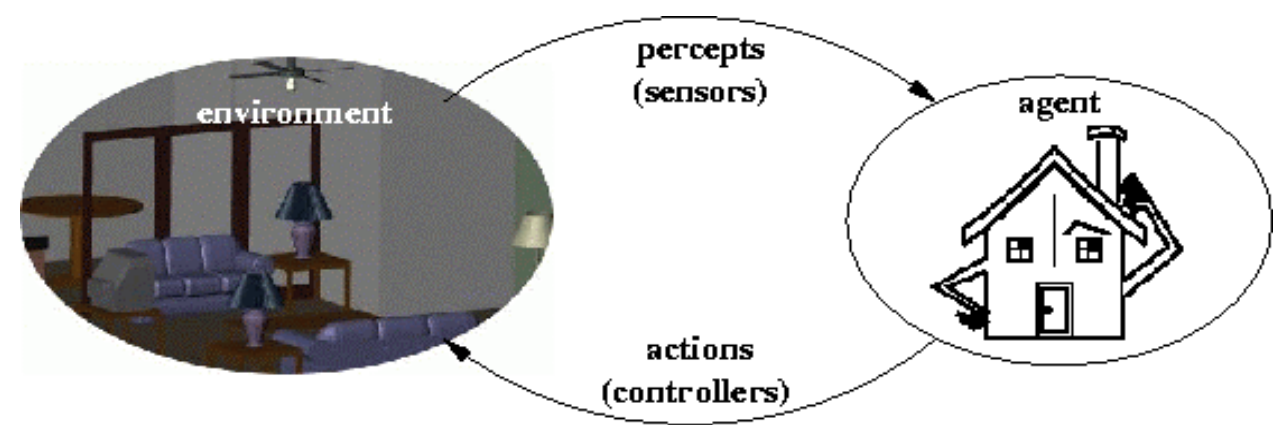

Fig. 1. Current directions in multi-agent research for smart environments.

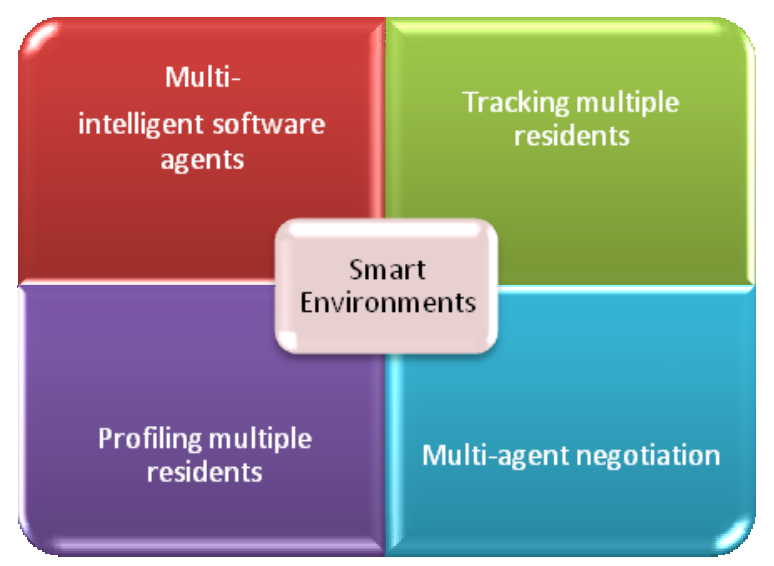

Fig. 2. Current directions in multi-agent research for smart environments.

viewed as an intelligent agent. In order to ensure that the collection of software agents is robust, transparent, and seamless, these embedded agents need to communicate and cooperate with each other.

A number of groups have investigated multiagent designs for smart environment architectures. When designing a multi-agent approaches, researchers need to consider 1) the software role each agent will assume, 2) the organization type that will exist between the agents, and 3) the method by which the agents will cooperate and will share information. For example, Duan and Li [8] have designed a multiagent system architecture for intelligent environments that utilizes a ZigBee wireless sensor network for communication. To address the agent organization issue, they define three layers of agents. Agents at the lowest layer are end-devices for the ZigBee network and connect with sensors and controllers. In the middle layer, each agent contributes a specific function such as identifying resident behaviors and preferences. Finally, the agent at the highest layer manages the total system.
In a similar fashion, the MavHome software architecture [23] also makes use of a hierarchical configuration of agents. Perception is performed in a bottom-up fashion: sensors in the bottom physical layer of the architecture monitor the environment and make readings available through the communication layer. The database stores this data in the information layer as more useful knowledge (e.g., patterns, predictions) that is made available as needed to the decision layer. In contrast, action execution originates at the top from the decision layer where the action is selected, the selected actions is stored in the information layer and sent through the communication layer to the physical components that execute the command. Communication between agents for this smart home is performed using CORBA and software agents register their presence using zero configuration technologies.

While the previous two approaches defined agent roles by functionality, and others define agent roles by device type (e.g., cell phone, PDA, electronic device) [19], alternative configurations have also been investigated. Agent roles can be defined by space, as has been explored by Chen and Tseng [4]. Space agents are defined for regions of the house and are deployed hierarchically, ultimately being controlled by Microsoft's Simple Control Protocol and Universal Plug and Play technology.

While many smart environment software agents rely on expert system or machine learning technology, the iDorm project [10] uses a collection of fuzzy agents to realize the vision of the smart environment. A nice complement to this project is the research of Acampora and Loia [1], who facilitate communication between agents using a Fuzzy Mark-Up Language. The multi-agent system distributes the fuzzy rules and variables to multiple hosts, facilitating the parallelization of fuzzy inference for a system such as a smart environment. 
These multi-agent software architectures support the design of complex smart environments. They also facilitate the scalability of such environments to large spaces with diverse functionalities. However, a challenge that has not been addressed much in the literature is how to adapt to and serve multiple residents in a smart environment. This challenge creates additional directions for multi-agent research that will be detailed in the following three sections of this article.

\section{Tracking multiple residents}

Once multiple residents enter a smart environment, the services provided by the smart environment become much more difficult to realize. Residents move and act differently when others are present. In addition, interaction between the residents is an entity of its own that may benefit from features that smart environments can provide.

The difficulties raised by multiple resident situations are reflected in the fact that few researchers have tackled these issues. A functionality that is required of many smart environments is the ability to find and track residents within the space. Tracking individual residents can be accomplished by looking at the history of motion sensor activations. When multiple residents enter the space, this is no longer a straightforward solution. There are simplifications that would ease the complexity of this task. For example, we could ask residents to wear devices that enable tracking them through the space $[11,22]$. This particular solution is impractical, however, for situations in which individuals do not want to wear the device, forget to wear the device, or enter and leave the environment frequently.

While tracking multiple residents is clearly a challenging problem and in fact has been proven to be NP-Hard [16], researchers are designing creative approaches to address the problem. Wen-Hau et al. [20], for example, have built pressure sensors into the floor of their smart environment that identify and locate each resident based on a weight profile. The approach does face the limitation of not being able to differentiate between individuals if they have close weight readings or are right next to each other in the space. Other researchers fuse multiple sources of information to track multiple residents in a space. As an example, Checka et al. [2] combine sound and vision to track multiple people. Information from these multi-modal sources is used to derive observation likelihoods. In this manner, not only are multiple information sources fused, but a confidence factor can be maintained for the computed identity and location of individuals within the smart environment.

\section{Behavior profiling for multiple residents}

Tracking the location of residents in a smart environment is a first step toward the goal of providing context-aware and location-aware services to the residents. However, it is not the end-all feature that is required of environments that act as intelligent agents. In order to provide services such as health monitoring and assistance, task automation, and analysis of activities, smart environments need to learn behavior profiles for all of the individuals that inhabit each environment.

Activity recognition is a well-investigated research issue. Researchers have successfully learned models for activity recognition from motion sensor data [14,21] wearable sensor data [15], and other wireless sensor information [18], albeit in singleresident settings. Very little work, however, has focused on learning behavior profiles for multiple residents.

An initial effort has been directed toward this goal of profiling multiple residents in a single environment setting. Specifically, Crandall and Cook [7] have designed machine learning approaches to attributing sensor events to the individual in the space that triggered the event. They have compare naïve Bayes and Markov model approaches to this classification problem as a precursor for learning entire activity profiles. Experimentation with data from a smart apartment indicates that the mapping of sensor events to residents is very doable - the naïve Bayes classifier performed best and achieved 98\% classification accuracy. However, this performance was achieved when the algorithm only considered motion sensor events with long durations. The researchers found that motion sensor events with short durations are indicative of residents transitioning between spaces and tasks, and are not as indicative of the person's location or activity as are events with longer durations.

\section{Multi-agent negotiation}

Finally, we consider the task of multi-agent negotiation. Whether the agents represent software entities, human residents, or software agents representing the interests of the human residents, effective negotiation strategies need to be design. Agents compete for re- 
sources and often have divergent, if not competing, needs and preferences.

Lin and Fu [14] learn a model of users' preferences that represents the relationships among users and the dependency between services and sensor observations. By explicitly learning relationships between these entities, their algorithm can automatically select the service that is appropriate for a given resident and context. Because their approach infers the best service at the right time and place, the comfort of residents can be maintained, even in a multiresident setting.

The competition for resources can be observed even at a software component level. In the work of Colley and Stacey [5], the need for software agents to communicate is recognized. Communication in a complex setting such as a smart environment can become quite costly, when the amount of gathered data and the number of provided services increases. In some circumstances it is advantageous for software agents to form clusters, or associate themselves with other agents with whom they will communicate often. Colley and Stacey investigate alternative voting schemes to design optimal clusters for this type of agent organization that meet an optimal number of needs and preference requests.

While the previous approaches have demonstrated success in negotiating needs of multiple agents in smart environments, there are many situations in which not all of the stated needs or preferences can be met. In response, the smart environment could prioritize the needs or the residents themselves. An alternative approach, put forward by Roy et al. [16], focuses not on meeting all of the needs of all of the agents, but instead to strike a balance between multiple diverse preferences. The goal of this approach is to achieve a Nash equilibrium state so that predictions and actions achieve sufficient accuracy in spite of possible correlations or conflicts. As demonstrated in this work, the result can be effective for goals such as minimizing energy usage in a smart environment.

\section{Challenges and directions for ongoing research}

In this paper we have provided an overview of current research in the area of multi-agent systems for smart environments. By no means do these efforts encompass all of the challenges and opportunities that researchers face in this area. Researchers who work in this field acknowledge that the problems of scalability are daunting. Scalability here can refer to the number of data sources and services that the smart environment will support. It can also refer to the number of residents that exist in the smart space. In both situations, novel approaches are needed to efficiently and effectively track, predict, and respond to the actions and needs of the agents in the space.

While the challenge of bringing smart environments to multi-agent scales is somewhat overwhelming, multi-agent settings also raise a number of opportunities for unique research. For example, the interactions between multiple residents in a smart environment sometimes take on a life of their own. Researchers may consider challenges such as learning social networks among residents in a smart environment from sensor data that is collected in the environment. They may also try to identify key classes of interactions such as parents playing with children or caregivers providing assistance to family members. The results of such efforts will provide key insights for smart environment systems that provide health monitoring and assistance.

Because smart environments and other ambient intelligent settings are extremely large and complex software systems, researchers do effectively compartmentalize the system components by delegating the work among multiple agents. These approaches are discussed in Section 2 of this paper. Interestingly, adopting a multi-agent software architecture for smart environments opens up a number of new research directions. If the agents originate from disparate sources then they may not use the same representation or approach to reasoning. A possible new research direction is to adapt belief-desire-intention for models to the study of agents in smart environments [2].

Another challenge for multi-agent smart environment software architectures is to define lightweight, simple, and scalable methods for communicating between the agents. While standards such as CORBA have traditionally filled this role, researchers have recently considered publish/subscribe approaches for communication [8]. Pub/sub middleware allows agents to asynchronously share information with little overhead. Continued research is needed to define efficient pub/sub communication approaches and languages for communicating among smart environment agents that utilize the features of pub/sub middleware methods.

Once interactions between multiple agents in a smart environment are identified, the smart environment can provide services that are unique for those interactions, such as emulating the reminder assistance that the caregiver provides. Researching and 
addressing such issues will allow smart environment research to move into new, agent-rich settings, such as smart airports, smart shopping malls, and smart hospitals.

\section{References}

[1] G. Acampora and V. Loia. Fuzzy control interoperability and scalability for adaptive domotic framework. IEEE Transactions on Industrial Informatics, 1(2):97-101, 2005.

[2] J.C. Augusto and J. O'Donoghue. The 6Ws architecture. To appear in the Proceedings of the International Conference on Agents and Artificial Intelligence, 2009.

[3] N. Checka, K. Wilson, M. Siracusa, and T. Darrell. Multiple person and speaker activity tracking with a particle filter. Proceedings of the International Conference on Acoustics, Speech, and Signal Processing, pages 881-884, 2004.

[4] W. Chen and W. Tseng. A novel multi-agent framework for the design of home automation. Proceedings of the International Conference on Information Technology, pages 277281, 2007.

[5] M. Colley and P. Stacey. Agent association group negotiation within intelligent environments. Proceedings of the International Conference on Intelligent Environments, 2008.

[6] D. Cook and S.K. Das. How smart are our environments? An updated look at the state of the art. Journal of Pervasive and Mobile Computing, 3(2):53-73, 2007.

[7] A. Crandall and D. Cook. Attributing events to individuals in multi-inhabitant environments. Proceedings of the International Conference on Intelligent Environments, 2008.

[8] G. Cugola and H.-A. Jacobsen. Using publish-subscribe middleware for mobile systems. ACM SIGMOBILE Mobile Computing and Communications Review, 6(4):25-33, 2002.

[9] P. Duan and H. Li. Zigbee wireless sensor network based multi-agent architecture in intelligent inhabited environments. Proceedings of the International Conference on Intelligent Environments, 2008.

[10] H. Hagras, V. Callaghan, M. Colley, G. Clarke, A. PoundsCornish, and H. Duman. Creating an ambient-intelligence environment using embedded agents. IEEE Intelligent Systems, 19(6):12-20, 2004.

[11] J. Hightower and G. Borriello. Location systems for ubiquitous computing. IEEE Computer, 32(8):57-66, August, 2001.
[12] J. Krumm, S. Harris, B. Meyers, B. Brumitt, M. Hale, and S Shafer. Multi-camera multi-person tracking for easy living. Proceedings of the IEEE International Workshop on Visual Surveillance, pages 3-10, 2000.

[13] Z. Lin and L. Fu. Multi-user preference model and service provision in a smart home environment. Proceedings of the IEEE International Conference on Automation Science and Engineering, pages 759-764, 2007.

[14] C. Lu, Y. Ho, and L. Fu. Creating robust activity maps using wireless sensor network in a smart home. Proceedings of the Third Annual Conference on Automation Science and Engineering, 2007.

[15] U. Maurer, A. Smailagic, D. Siewiorek, and M. Deisher. Activity recognition and monitoring using multiple sensors on different body positions. Proceedings of the International Workshop on Wearable and Implantable Body Sensor Networks, 2006.

[16] N. Roy, A. Roy, and S. K. Das. Context-aware resource management in multi-inhabitant smart homes: A Nash h-learning based approach. Proceedings of the IEEE International Conference on Pervasive Computing and Communications, pages 148-158, July 2006.

[17] S. Russell and P. Norvig. Artificial Intelligence: A Modern Approach. Prentice Hall, second edition, 2002.

[18] D. Sanchez, M. Tentori, and J. Favela. Activity recognition for the smart hospital. IEEE Transactions on Systems, Man and Cybernetics, Part A: Systems and Humans, 35:133-144, 2005.

[19] J. Velasco, I. Maestre, A. Navarro, M. López, A. Vicente, E. de la Hoz, A. Paricio, and M. Machuca. Location-aware services and interfaces in smart homes using multiagent systems. Proceedings of the International Conference on Pervasive Systems and Computing, pages 104-110, 2005.

[20] L. Wen-Hau, W. Chao-Lin, and F. Li-Chen. Inhabitants tracking system in a cluttered home. IEEE Transactions on Automation Science and Engineering, 5(1):10-20, 2008.

[21] C.R. Wren and E.M. Tapia. Toward scalable activity recognition for sensor networks. Proceedings of the International Workshop on Location- and Context-Awareness, 3987:168$185,2006$.

[22] J. Yin, Q. Yang, and D. Shen. Activity recognition via usertrace segmentation. ACM Transactions on Sensor Networks, November, 2008.

[23] G.M. Youngblood and D. Cook. Data mining for hierarchical model creation. IEEE Transactions on Systems, Man, and Cybernetics, Part C, 37(4):561-572, 2007. 